P57 (continued)

macronutrients, micronutrients, critical thinking skills, and designing and analyzing diets. Strategies identified to ease understanding of difficult concepts included additional exposure to concepts with interactive materials and more study time.

Conclusions and Implications: Results suggest additional strategies are needed when covering digestion and metabolism concepts in introductory nutrition courses. Covering fewer concepts more in-depth with an interactive approach to allow additional exposure may be warranted.

Funding: None

\section{P58 The Ability of the Registered Dietitian to Promote Weight Gain Prevention}

Catherine Metzgar, RD, LDN, metzgar2@illinois.edu, University of Illinois at Urbana-Champaign, 260 Bevier Hall, 905 S. Goodwin, Urbana, IL 61801;

S. M. Nickols-Richardson, PhD, RD

Objective: To compare the ability of the registered dietitian with a counselor to facilitate weight gain prevention. Target Audience: Young adult and perimenopausal women.

Theory, Prior Research, Rationale: Intervention efforts to prevent the development of obesity should target young adult and midlife women as these are crucial intervals for weight gain. Few studies have addressed weight gain prevention and none have compared the facilitation of weight management by a registered dietitian against another health professional. This study will be grounded in Social Cognitive Theory.

Description: Women $(n=180)$ will be recruited for this 12-month parallel-arm weight gain prevention randomized controlled trial. Women will be randomized to a nutrition education intervention group led by a registered dietitian (RDG) or counselor (CSG), or a wait-list control $(\mathrm{CON})$ group. Women in the intervention groups will attend identical education sessions that cover general nutrition and fitness information and emphasize portion control, planning ahead for food intake and vegetable consumption. The only difference will be the leader of the intervention (RDG vs. CSG).

Evaluation: The main outcome will be change in body weight. Changes in blood pressure, biochemical markers of metabolism, dietary intake, physical activity participation and Social Cognitive Theory mediators of behavior will be assessed.

Conclusions and Implications: Compared to the CON group, women in the intervention groups are hypothesized to maintain current body weight; women in the RDG group are postulated to have the least weight gain over time. Findings will help inform the role of the registered dietitian in weight gain prevention and provide a foundation for future weight gain prevention studies.

Funding: None
P59 Systematic Review of Culinary Interventions to Promote Healthy Eating: Directions for Future Research and Practice

Henna Muzaffar,PhD, RD, LDN, muzaffa1@uiuc.edu, University of Illinois at Urbana-Champaign, $904 \mathrm{~W}$. Nevada Street, MC-081, Urbana, IL 61801; B. Fiese, PhD; J. M. Jarick, BS

Objective: A systematic review was conducted to evaluate culinary skills interventions to identify specific programs and programmatic factors associated with improvement in the quality of diet, BMI, and positive changes in psychosocial variables.

Target Audience: The settings for culinary interventions included schools, colleges and the community. Target population included children, adolescents, and adults.

Theory, Prior Research, Rationale: Policy makers, scientists, and food and nutrition practitioners suggest that there is a societal decline in culinary skills, which is predictive of poor dietary habits contributing to childhood obesity. Twelve interventions were grounded in a behavioral theory and 7 incorporated social cognitive theory framework.

Description: Pubmed/Medline, Cochrane database and hand search identified 131 articles, 26 articles were selected for further examination based on inclusionary criteria. Study designs included 2 randomized controlled trials, 4 quasi-experimental, 1 non-randomized pre and post comparison study, and 19 pilot studies. Study methodology included cooking classes, nutrition education lessons, parent and community components, gardening classes, school lunchroom components, tasting sessions, and trips to farmers market.

Evaluation: Qualitative evaluations of the programs indicate positive findings in terms of program appeal and improvement in cooking skills and healthy eating. Quantitative analysis indicate improvement in food preferences, cooking skills, cooking self-efficacy, cooking behavioral intentions, food preparation frequency, knowledge, healthy dietary intake, BMI, and blood pressure.

Conclusions and Implications: The findings from this review support a positive relationship between culinary interventions and improvement in cooking skills, consumption of a healthy diet and positive changes in anthropometric assessments. However, further research should emphasize rigorous methodological standards, develop theory-based standardized frameworks and evaluate longterm effects of interventions.

Funding: None

\section{P60 Pre-Health Professionals' Attitudes About Obesity Before and After an Obesity and Weight Management Course}

Lori Klos,PhD, RD, neighbol@uwm.edu, University of Wisconsin-Milwaukee, Department of Kinesiology, 455 Enderis Hall, Milwaukee, WI 53201; C. Greenleaf, PhD; T. Cass; N. Paly, BS; M. Kessler, BS 